\title{
BMJ Open Predicting muscle loss during lung cancer treatment (PREDICT): protocol for a mixed methods prospective study
}

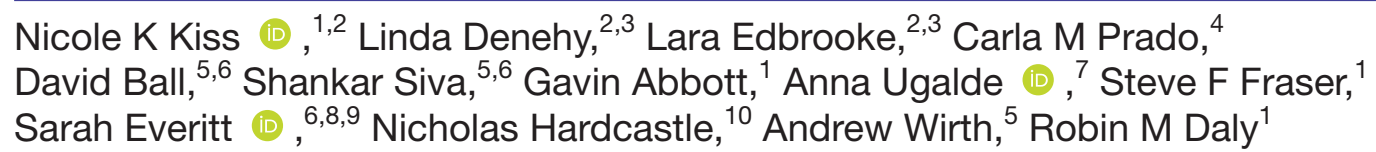

To cite: Kiss NK, Denehy L, Edbrooke L, et al. Predicting muscle loss during lung cancer treatment (PREDICT): protocol for a mixed methods prospective study. BMJ Open 2021;11:e051665. doi:10.1136/ bmjopen-2021-051665

- Prepublication history and additional supplemental material for this paper are available online. To view these files, please visit the journal online (http://dx.doi.org/10.1136/ bmjopen-2021-051665).

Received 24 March 2021 Accepted 06 September 2021

Check for updates

(C) Author(s) (or their employer(s)) 2021. Re-use permitted under CC BY-NC. No commercial re-use. See rights and permissions. Published by BMJ.

For numbered affiliations see end of article.

Correspondence to

Dr Nicole K Kiss;

nicole.kiss@deakin.edu.au

\section{ABSTRACT}

Introduction Low muscle mass and low muscle attenuation (radiodensity), reflecting increased muscle adiposity, are prevalent muscle abnormalities in people with lung cancer receiving curative intent chemoradiation therapy (CRT) or radiation therapy (RT). Currently, there is a limited understanding of the magnitude, determinants and clinical significance of these muscle abnormalities in the lung cancer CRT/RT population. The primary objective of this study is to identify the predictors of muscle abnormalities (low muscle mass and muscle attenuation) and their depletion over time in people with lung cancer receiving CRT/RT. Secondary objectives are to assess the magnitude of change in these parameters and their association with health-related quality of life, treatment completion, toxicities and survival.

Methods and analysis Patients diagnosed with lung cancer and planned for treatment with CRT/RT are invited to participate in this prospective observational study, with a target of 120 participants. The impact and predictors of muscle abnormalities (assessed via CT at the third lumbar vertebra) prior to and 2 months post CRT/RT on the severity of treatment toxicities, treatment completion and survival will be assessed by examining the following variables: demographic and clinical factors, weight loss, malnutrition, muscle strength, physical performance, energy and protein intake, physical activity and sedentary time, risk of sarcopenia (Strength, Assistance in walking, Rise from a chair, Climb stairs, Falls history (SARC-F) score alone and with calf-circumference) and systemic inflammation. A sample of purposively selected participants with muscle abnormalities will be invited to take part in semistructured interviews to understand their ability to cope with treatment and explore preference for treatment strategies focused on nutrition and exercise.

Ethics and dissemination The PREDICT study received ethics approval from the Human Research Ethics Committee at Peter MacCallum Cancer Centre (HREC/53147/PMCC-2019) and Deakin University (2019320). Findings will be disseminated through peer review publications and conference presentations.

\section{INTRODUCTION}

Over two million new cases of lung cancer were diagnosed globally in 2018, with lung cancer the leading cause of death from

\section{Strengths and limitations of this study}

- This is a multisite study across three tertiary health services with specialist cancer services.

- Study outcomes are assessed using tools and techniques with established validity in the field.

- The use of tools and techniques that are widely used within clinical practice enhances the applicability of findings to future practice.

- Due to the observational study design, causality for muscle abnormalities cannot be established from the findings

- Radiological data will be acquired from multiple scanners with consistent protocols and quality control in place.

cancer worldwide accounting for almost $20 \%$ of cancer mortality. ${ }^{1}$ The most common curative intent treatment for locally advanced non-small cell lung cancer (NSCLC) and limited stage small cell lung cancer (SCLC) is a lengthy and demanding course of chemoradiation therapy (CRT). CRT can be associated with severe acute toxicities and significant nutritional and functional decline related to muscle loss, a key feature of malnutrition and cancer cachexia. ${ }^{2}$ Median survival following curative CRT is less than 2.5 years. ${ }^{3}$ However, advances in immunotherapy are promising and demonstrate capacity to improve survival following definitive CRT. ${ }^{4}$ Such advances highlight the increasing importance of ensuring that patients who complete CRT are in optimal physical condition to withstand the lengthy and demanding treatments.

The use of routine CT images has emerged as an opportunistic way to quantify muscle mass and assess muscle attenuation. ${ }^{5}{ }^{6}$ Low muscle attenuation (radiological density measured in Hounsfield Units (HU)) is reflective of increased intermuscular adiposity. ${ }^{7}$ A number of studies have demonstrated the negative consequences associated 
with muscle abnormalities in people with cancer. ${ }^{589}$ Low muscle mass prior to treatment is highly prevalent (up to $47 \%$ ) in patients with stage III and IV NSCLC treated with chemotherapy and is independently associated with up to a twofold increased risk of mortality. ${ }^{10}{ }^{11}$ In colorectal cancer, muscle loss of $9 \%$ or more during chemotherapy has been independently associated with an almost fivefold higher risk of shorter survival. ${ }^{12}$ Several studies have also found low muscle mass to be predictive of chemotherapyrelated toxicity in multiple cancer types. ${ }^{813}{ }^{14}$ Likewise, low muscle attenuation has been associated with reduced survival in patients with colorectal and renal cell cancers, increased inflammation and impaired muscle function in non-cancer populations. ${ }^{121516}$ To date, most research in patients with lung cancer treated with chemotherapy has focused on the prognostic impact of muscle abnormalities. Little is known about the magnitude of muscle loss and degree of muscle fat infiltration (attenuation) and their impact on treatment outcomes, function, quality of life or the experience of people with lung cancer treated with CRT or radiation therapy (RT). As the most common treatment modality for stage III NSCLC and limited stage SCLC, understanding the prevalence, magnitude and consequences of muscle abnormalities in patients treated with CRT/RT is of significant clinical importance. Furthermore, it can inform targeted nutrition and exercise interventions to improve outcomes given that there are currently no pharmaceutical agents available to counteract muscle abnormalities.

Complicating the identification of low muscle mass is the increasing prevalence of patients presenting with overweight or obesity, often masking the presence of low muscle mass. Although approximately $50 \%$ of patients with advanced lung cancer present with excess body weight, low muscle mass is present across all body mass index (BMI) categories. ${ }^{51718}$ Current nutrition assessment tools misclassify up to $60 \%$ of patients as well-nourished when they have low muscle mass defined on CT, meaning they may be overlooked for nutrition intervention. ${ }^{19}$ Consequently, alternate methods are required to identify people presenting with adverse body composition changes such as low muscle mass at treatment commencement or experiencing muscle loss during treatment.

In a preliminary study in 41 patients with NSCLC, we found that $61 \%$ had low muscle mass before commencing CRT, despite $61 \%$ having overweight/obesity. ${ }^{20}$ We also identified that $41 \%$ had low muscle attenuation, which has been linked to increased inflammation and impaired muscle function. ${ }^{16}$ Further, loss of muscle mass occurred in over half the patients by 3 months following CRT. ${ }^{20}$ Building on this work, the primary aim of this study is to identify the predictors of muscle abnormalities (low muscle mass and low muscle attenuation) and their loss relative to treatment commencement in people with lung cancer following curative intent CRT/RT. In addition, the magnitude of change in muscle mass and muscle attenuation, and associations of muscle abnormalities and changes in muscle mass or muscle attenuation with health-related quality of life (HR-QoL), treatment outcomes and survival, along with patient experience of living with muscle abnormalities, will be examined.

\section{METHODS AND ANALYSIS}

This mixed methods prospective study is recruiting at three tertiary hospitals in Victoria, Australia. The study is conducted by researchers and clinicians at Deakin University, Peter MacCallum Cancer Centre, University of Melbourne and University of Alberta.

\section{Study population}

Patients who are candidates for curative intent standard dose and fraction CRT/RT for a confirmed diagnosis of NSCLC or SCLC (any disease stage) are approached to participate in the study. Patients will receive volumetric modulated arc therapy with online cone-beam CT (CBCT) image guidance. Patients will be simulated and treated in free-breathing, without respiratory gating techniques. Simulation imaging involves acquisition of a four-dimensional CT, on which a gross tumour volume (GTV) will be segmented. The GTV on each phase of the respiratory cycle will be combined to form the internal GTV (iGTV). A $5 \mathrm{~mm}$ margin will be applied to the iGTV to obtain the clinical target volume, followed by an additional $1 \mathrm{~cm}$ margin to obtain the planning target volume. Adaptive radiotherapy is not routinely performed but may be considered in the case of significant anatomical change or tumour shrinkage as indicated on CBCTs acquired during treatment. Concurrent chemotherapy includes cisplatin/paclitaxel, cisplatin/etopiside, cisplatin/pemetrexed, carboplatin/paclitaxel, carboplatin/etopiside. Eligible patients are identified through screening clinic lists and discussion with the lung multidisciplinary team at each health service. Eligibility criteria are described in table 1. Non-English speaking patients are eligible to participate if they can provide informed consent and participate in the study with the aid of an interpreter or family member. Recruitment commenced in September 2019 was suspended from March to October 2020 due to the COVID-19 pandemic and recommenced in November 2020 .

\begin{tabular}{ll}
\hline Table $1 \quad$ Study eligibility criteria \\
\hline Inclusion criteria & Exclusion criteria \\
\hline Aged $\geq 18$ years & $\begin{array}{l}\text { Patients with a cognitive } \\
\text { impairment or psychiatric illness } \\
\text { reported in the medical history }\end{array}$ \\
$\begin{array}{l}\text { Planned for standard dose } \\
\text { and fraction radiotherapy } \\
\text { regimens, that is, not } \\
\text { stereotactic ablative body } \\
\text { radiotherapy (SABR) }\end{array}$ & $\begin{array}{l}\text { Conditions known to affect body } \\
\text { composition including HIV, recent } \\
\text { diagnosis of thyroid disease, } \\
\text { muscular dystrophy or other } \\
\text { neurodegenerative conditions }\end{array}$ \\
$\begin{array}{l}\text { Have CT image available } \\
\text { within } 30 \text { days of the } \\
\text { baseline study assessment }\end{array}$ & \\
\hline
\end{tabular}




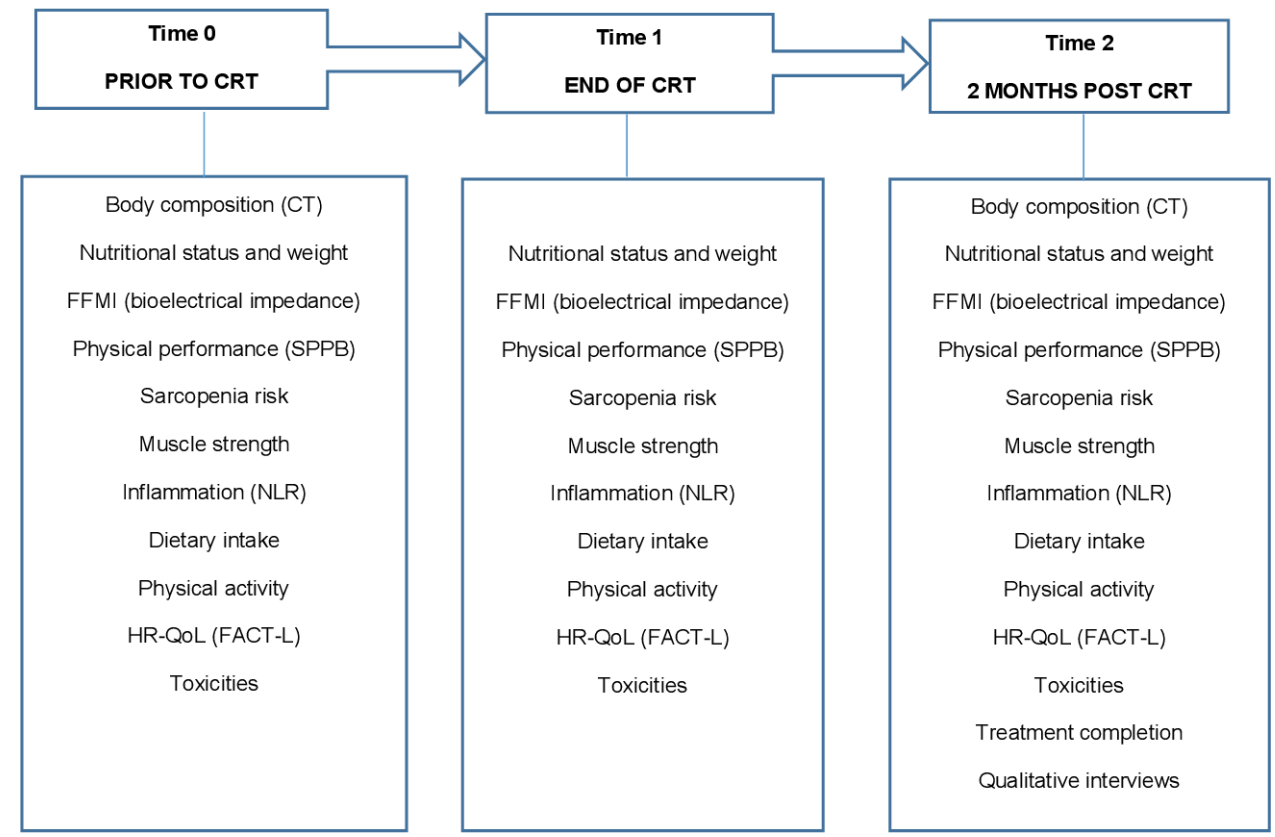

Figure 1 Study schema describing data collection time points and study assessments. CRT, chemoradiation therapy; FACT-L, functional assessment of cancer therapy_lung; FFMI, fat-free mass index; HR-QoL, health-related quality of life; NLR, neutrophil to lymphocyte ratio; SPPB, short physical performance battery.

Baseline data (T0) are collected prior to or within 1 week of commencing CRT/RT (figure 1). Follow-up data collection occurs at the end of CRT/RT (T1) and at 2 months following completion of CRT/RT (T2).

\section{Participant and treatment characteristics}

Demographic data on age, sex, living situation (alone or with others), level of education, smoking status, Charlson comorbidity score, medications, disease staging and treatment plan are collected at baseline from participants' medical records. Radiotherapy dose, volume (cc), location (left upper lobe, right upper lobe, right lower lobe, left lower lobe), and mean and maximum oesophageal dose are recorded from the radiotherapy treatment plan.

\section{Measures}

Data on CT derived muscle mass, muscle attenuation and visceral and subcutaneous adipose tissue, nutritional status, weight, bioimpedance spectroscopy fatfree mass and fat mass, physical performance, muscle strength, inflammation, dietary intake, physical activity and sedentary time, toxicities and HR-QoL are collected as described in the study schema (figure 1). Data on treatment completion are collected at the completion of CRT/RT and survival data will be censored at 12 months following recruitment of the final participant. Table 2 provides an overview of all study assessments.

\section{Anthropometry, muscle mass and body composition}

Weight is measured on a commercially available floor scale to the nearest $0.1 \mathrm{~kg}$ (Tanita Innerscan 50, Tanita, Australia). Height is obtained from the medical record. Calf-circumference is measured to the nearest $0.1 \mathrm{~cm}$ three times and the mean value recorded. Participants are asked to self-report their weight at 6 and 12 months prior to each assessment.

Muscle mass, muscle attenuation, visceral, subcutaneous and intramuscular adipose tissue at the third lumbar vertebra (L3) are assessed from routine CT images taken for diagnostic or staging purposes (T0) and treatment evaluation (T2) using the Alberta protocol and the date of the image recorded. ${ }^{11}{ }^{21}$ In brief, muscle attenuation measured in HU and skeletal muscle crosssectional area $\left(\mathrm{CSA}, \mathrm{cm}^{2}\right)$ are measured by a specifically trained observer from a single cross-sectional axial image L3 using SliceOmatic software (V.5.0, Tomovision, Quebec, Canada). L3 is the standard landmark that correlates best with whole body muscle mass, ${ }^{621}$ and contains the psoas, paraspinal muscles (erector spinae, quadratus lumborum) and abdominal wall muscles (transversus abdominis, external and internal obliques, rectus abdominis). Total muscle CSAs, derived from the combined area of each of these individual muscles, are identified within an HU range of -29 to $150^{22}$ and normalised for height $\left(\mathrm{m}^{2}\right)$ to determine skeletal muscle index (SMI, $\mathrm{cm}^{2} / \mathrm{m}^{2}$ ). Muscle attenuation is measured as the mean HUs within all the segmented muscles at L3. BMI and sex-specific cut-off points are used to identify low total muscle mass and low total muscle attenuation according to published definitions:

Low SMI (representing muscle mass) defined as $<43 \mathrm{~cm}^{2} / \mathrm{m}^{2}$ in men with a BMI $<24.9 \mathrm{~kg} / \mathrm{m}^{2},<53 \mathrm{~cm}^{2} /$ $\mathrm{m}^{2}$ in men with a BMI $\geq 25 \mathrm{~kg} / \mathrm{m}^{2}$, and $<41 \mathrm{~cm}^{2} / \mathrm{m}^{2}$ in women of any BMI. ${ }^{5}$

Low muscle attenuation defined as $<41 \mathrm{HU}$ in men and women with a BMI $<24.9 \mathrm{~kg} / \mathrm{m}^{2}$, and $<33 \mathrm{HU}$ in men and women with $\mathrm{BMI} \geq 25 \mathrm{~kg} / \mathrm{m}^{2}$. 
Table 2 Study measures

\begin{tabular}{|c|c|c|c|c|}
\hline Study measure & Instrument & TO & T1 & T2 \\
\hline \multicolumn{5}{|l|}{ Potential predictors } \\
\hline Malnutrition & PG-SGA & $\checkmark$ & $\checkmark$ & $\checkmark$ \\
\hline Muscle strength & Handgrip dynamometer & $\checkmark$ & $\checkmark$ & $\checkmark$ \\
\hline Bioimpedence spectroscopy & $\begin{array}{l}\text { Total body and appendicular } \\
\text { (arms+legs) fat-free mass, fat mass }\end{array}$ & $\checkmark$ & $\checkmark$ & $\checkmark$ \\
\hline $\begin{array}{l}\text { Physical activity and sedentary } \\
\text { behaviour }\end{array}$ & $\begin{array}{l}\text { Accelerometer, inclinometer, IPAQ- } \\
\text { short form }\end{array}$ & $\checkmark$ & $\checkmark$ & $\checkmark$ \\
\hline Physical performance & SPPB & $\checkmark$ & $\checkmark$ & $\checkmark$ \\
\hline Sarcopenia risk & SARC-F, calf-circumference & $\checkmark$ & $\checkmark$ & $\checkmark$ \\
\hline Protein and energy intake & 3-day food record & $\checkmark$ & $\checkmark$ & $\checkmark$ \\
\hline Systemic inflammation & Neutrophil to lymphocyte ratio & $\checkmark$ & $\checkmark$ & $\checkmark$ \\
\hline \multicolumn{5}{|l|}{ Primary outcome } \\
\hline Muscle mass & CT & $\checkmark$ & $x$ & $\checkmark$ \\
\hline \multicolumn{5}{|l|}{ Secondary outcomes } \\
\hline Muscle attenuation & CT & $\checkmark$ & $\mathrm{x}$ & $\checkmark$ \\
\hline $\begin{array}{l}\text { Visceral, subcutaneous and } \\
\text { intramuscular adipose tissue }\end{array}$ & & $\checkmark$ & $\mathrm{x}$ & $\checkmark$ \\
\hline Treatment completion & Medical record & $x$ & $x$ & $\checkmark$ \\
\hline Toxicities & CTCAE V.5.0 & $\checkmark$ & $\checkmark$ & $\checkmark$ \\
\hline Survival & Medical record or death registry & $12 r$ & ecru & \\
\hline
\end{tabular}

CTCAE, Common Terminology Criteria for Adverse Events; IPAQ, International Physical Activity Questionnaire-short form; PG-SGA, PatientGenerated Subjective Global Assessment; SARC-F, Strength, Assistance in walking, Rise from a chair, Climb stairs, Falls history; SPPB, short physical performance battery.

Subcutaneous adipose tissue CSA $\left(\mathrm{cm}^{2}\right)$ is identified within a $\mathrm{HU}$ range of -190 to $-30 .^{22}$ Visceral adipose tissue CSA $\left(\mathrm{cm}^{2}\right)$ is identified within a HU range of -150 to $-50 .^{23}$ Intramuscular adipose tissue CSA $\left(\mathrm{cm}^{2}\right)$ is identified within a HU range of -190 to $-30 .{ }^{22}$ The CSAs of visceral and subcutaneous adipose tissue are combined to derive total abdominal adipose tissue and normalised for height to determine the total adipose tissue index $\left(\mathrm{cm}^{2} /\right.$ $\mathrm{m}^{2}$ ). Where possible, we will minimise variation between images used for CT assessments; this includes selection for analysis of the same intravenous contrast phase, reconstruction filter (soft tissue) and slice thickness. Finally, segmented structures are manually performed by an expert observer therefore will be subject to quality control during segmentation process. ${ }^{24} 25$

Tetrapolar bioimpedance spectroscopy (SOZO, Impedimed, USA) is used to estimate total body and appendicular (arms and legs) fat-free mass and fat mass (all in $\mathrm{kg}$ ), total body water, extracellular and intracellular fluid and phase angle (ratio of resistance to reactance) using proprietary software provided by Impedimed (Brisbane, Australia). Participants are asked to stand on the SOZO scale, placing feet and hands on the corresponding foot and hand sensors.

\section{Nutritional status}

Nutritional status, that is, the presence or absence of malnutrition, is determined through the Patient- Generated
Subjective Global Assessment (PG-SGA). ${ }^{26}$ The PG-SGA is a common nutrition assessment tool used by oncology dietitians in clinical practice and categorises patients as: A-well-nourished, B-mild to moderate malnutrition, C-severe malnutrition. The PG-SGA has been evaluated as an outcome measure in clinical nutrition studies and validated for use in oncology patients undergoing radiotherapy. ${ }^{27} 28$ Data collected will also allow determination of a diagnosis of malnutrition using the Global Leadership on Malnutrition criteria. ${ }^{29}$

\section{Protein and energy intake}

Protein and energy intake is assessed using 3-day food records which are suitable for capturing total dietary intake over the short term. ${ }^{30}$ The 3 -day period has been chosen in order to account for day-to-day variation in dietary intake including 2 weekdays and 1 weekend day. ${ }^{31}$ Food records will be entered into the nutritional analysis tool, ASA-24-Australia. Individual participant energy and protein requirements will be estimated using standard equations recommended by the European Society for Clinical Nutrition and Metabolism guidelines on nutrition in cancer patients: 25-30 kilocalories/kg body weight, 1.0-1.5 g protein/kg body weight $^{32}$ to determine the proportion of energy and protein requirements met. 
Physical performance, muscle strength, sarcopenia risk

Physical performance is assessed using the short physical performance battery (SPPB). The SPPB consists of three objective measures used to score each of static balance, gait speed (over $4 \mathrm{~m}$ ) and lower body muscle strength (five times sit-to-stand test). The three objective measures are each scored from 0 to 4 , and combined to derive a total SPPB score ranging from 0 to 12, with higher scores indicating better performance. The SPPB has been demonstrated to have predictive validity for survival among cancer survivors. ${ }^{33}$ Upper limb muscle strength is assessed using the valid and reliable handgrip strength test which measures volitional grip force $(\mathrm{kg})$ applied by the combined contraction of extrinsic and intrinsic hand muscles. ${ }^{34}$ Handgrip strength is measured using a digital hand dynamometer (Jamar Digital Plus) following the standard protocol of the American Society of Hand Therapists. $^{35}$ In a seated position, $90^{\circ}$ elbow flexion and forearm mid-prone, participants will be required to apply as much force as possible to the dynamometer for $3-5 \mathrm{~s}$ using the right and then left hand. Participants complete one practice test followed by three tests on each hand (with a 5 s rest interval between tests), with the maximum strength from either hand used in the analysis. Grip strength of $<27 \mathrm{~kg}$ for men and $<16 \mathrm{~kg}$ for women is considered impaired. ${ }^{36}$ Sarcopenia risk is assessed using the SARC-F (Strength, Assistance in walking, Rise from a chair, Climb stairs, Falls history) tool alone and in combination with calf-circumference. The SARC-F is a rapid screening tool for sarcopenia in older adults. Sarcopenia is defined as a decline in muscle mass, strength and/or function. ${ }^{37}$ The SARC-F assesses five components including strength, assistance in walking, ability to rise from a chair, ability to climb stairs and occurrence of falls in the past year. The five components are scored from 0 to 2, and combined to derive a total SARC-F score ranging from 0 to 10 , with a score $\geq 4$ predictive of sarcopenia. ${ }^{37}$ Calf-circumference $<34 \mathrm{~cm}$ in men and $<33 \mathrm{~cm}$ in women is considered low. ${ }^{38}$ When used in combination with SARC-F, low calf-circumference is assigned an additional 10 points with a total score of $\geq 11$ predictive of sarcopenia. $^{38}$

The proposed cut-points that represent low muscle mass and low muscle strength will be reviewed prior to statistical analysis due to rapid, ongoing research in this field.

\section{Physical activity}

Daily sedentary time, light and moderate to vigorous physical activity are assessed over a 7-day period using an accelerometer attached by a belt at the hip level (ActiGraph wGT3X-BT, ActiGraph LLC, USA). The accelerometer measures accelerations at the hip (counts/min) in order to determine the time spent in sedentary, light, moderate or vigorous intensity activity based on sedentary $(<100$ counts/min), light (100-1951 counts/min), moderate (1952-5724 counts/min) and vigorous ( $\geq 5725$ counts/ min) intensity activity. ${ }^{3940}$ Participants are asked to keep a record of their sleep/awake times, periods of removal of the accelerometer (if any), naps taken (if any) and other relevant information. Step count and time spent sitting and standing are estimated using an inclinometer, which is attached to the participant's right thigh with a hypoallergenic patch (activPAL3, PAL Technologies, Glasgow, UK). The activPAL monitor has been demonstrated as valid and reliable in adults ${ }^{41}$ and older adults. ${ }^{42}$ Participants are instructed to wear the devices continuously (24 hours/day) for the 7-day period. Data for a minimum of any 4 valid days, with a valid day considered as 8 or more hours of wear time, will be required for analysis for both devices. ${ }^{43}$ Devices are applied with education provided by the trial research staff at the end of each outcome assessment.

Patient-reported baseline physical activity is assessed using the International Physical Activity QuestionnaireShort Form (IPAQ) at T0. The IPAQ is also completed at $\mathrm{T} 1$ and T2 as a source of patient-reported physical activity during and post-treatment. The IPAQ allows participant activity levels to be categorised as low, moderate or high based on the IPAQ scoring protocol. The IPAQ has been validated in young, middle age and older adults and is used in lung cancer populations. ${ }^{445}$

\section{Health-related quality of life}

HR-QoL is measured using the Functional Assessment of Cancer Therapy-Lung (FACT-L) Scale, a self-report instrument designed and validated for use in patients with lung cancer. ${ }^{46}{ }^{47}$ FACT-L consists of 27 core items (FACTGeneral) to assess patient function in four domains: physical, social/family, emotional and functional well-being. These domains are supplemented by a 9-item subscale used to assess symptoms specific to lung cancers (LCS). Higher scores indicate a better HR-QoL. Scores for Global Quality of Life, Physical Well-being (PWB), Functional Well-being (FWB), LCS and the Trial Outcome Index (sum of PWB, FWB and symptom subscale) will be determined.

\section{Inflammation}

Systemic inflammation is assessed using the neutrophil to lymphocyte ratio (NLR) which is collected from pathology reports in the participant medical record from routine blood tests taken throughout CRT. NLR is an easily measured, reproducible and inexpensive marker of subclinical inflammation. NLR is calculated by dividing the absolute neutrophil count by the absolute lymphocyte count, participants with a ratio $>3.53$ will be considered to have the presence of inflammation. ${ }^{48}$

\section{Treatment toxicity, treatment completion and survival}

The presence and severity of treatment toxicities, including dysphagia/oesophagitis, nausea and vomiting, is assessed using the Common Terminology Criteria for Adverse Events V.5.0 (CTCAE, NCI, USA) by the trial research staff. Interruptions to radiotherapy and the prescribed and delivered dose of radiotherapy are recorded from participants' medical 
Table 3 Common treatment regimens used for curative intent (chemo)radiotherapy treatment for non-small cell and small cell lung cancer

\begin{tabular}{|c|c|}
\hline Diagnosis & Treatment \\
\hline NSCLC & $\begin{array}{l}\text { Radiotherapy } 60 \text { Gy in } 30 \text { fractions plus platinum- } \\
\text { based chemotherapy* } \\
\text { Radiotherapy } 55 \text { Gy in } 20 \text { fractions without } \\
\text { chemotherapy }\end{array}$ \\
\hline SCLC & $\begin{array}{l}\text { Radiotherapy } 45 \text { Gy in } 30 \text { fractions plus platinum- } \\
\text { based chemotherapy* } \\
\text { Radiotherapy } 40 \text { Gy in } 15 \text { fractions plus platinum- } \\
\text { based chemotherapy* }\end{array}$ \\
\hline
\end{tabular}

${ }^{*}$ Cisplatin/paclitaxel, cisplatin/etopiside, cisplatin/pemetrexed, carboplatin/paclitaxel, carboplatin/etopiside.

NSCLC, non-small cell lung cancer; SCLC, small cell lung cancer.

records and confirmed with the treating team. Modifications to prescribed chemotherapy regimen, including dose reduction, early termination of chemotherapy or alteration to planned chemotherapy agents, are recorded from participants' medical records and confirmed with the treating team. Commencement of sequential immunotherapy within 3 months of completing CRT is recorded from participants' medical records and confirmed with the treating team. Table 3 describes the common treatment regimens. Overall survival time (months) will be calculated from the date of the baseline pre-CRT CT scan until death from any cause or the date of censoring at 12 months following recruitment of the final participant. Participant survival will be determined from the hospital medical record or death registry.

\section{Patient interviews}

A purposively selected sample of participants, who had low muscle mass at $\mathrm{T} 0$ or $\mathrm{T} 2$ and/or experienced any muscle loss at T2, are invited to participate in an interview following completion of T2 data collection. Interviews seek to understand their ability to cope with treatment and complete selfcare, receptiveness to nutrition and exercise intervention, when and in what form (individualised, group, in-person, technology-supported) they would prefer to receive nutrition and exercise intervention (online supplemental file 1). Where possible, diversity in participant demographics (age, sex, BMI, impaired function and living situation) is sought. It is anticipated that approximately 15-20 interviews will be required to reach data saturation. ${ }^{49}$

\section{Sample size}

A recruitment target of 120 patients over 32 months is based on treatment of 90 patients with CRT annually and a participation rate of $50 \%$ indicated by previous studies at these sites.$^{50}$ Power calculations for the primary objective are based on the strength of associations between individual predictors and the outcomes in the population that could be detected with $80 \%$ power given the expected size of analysis samples. With an initial sample of 120, the expected analysis samples at end of CRT and 2 months post-CRT are 105 and 84, allowing for $12.5 \%$ and $30 \%$ dropout, respectively. ${ }^{50}$ These sample sizes will provide $80 \%$ power to detect effect sizes of $r^{2}=0.08$ and 0.10 (ie, $8 \%$ and $10 \%$ of the outcome variance explained in a simple linear regression model), at T1 and $\mathrm{T} 2$, respectively, with $\alpha=0.05$. In a multiple linear regression model including up to eight covariates, sample sizes of 105 and 84 will provide $80 \%$ power to detect squared partial correlations of 0.07 and 0.09 , respectively, with $\alpha=0.05$.

Based on our previous research, ${ }^{20}$ we estimate (a) that the population HR is 1.87 for low muscle attenuation compared with normal muscle attenuation at baseline, (b) the proportion of the target population with low muscle attenuation will be $41 \%$ and (c) survival of $50 \%$ in the normal muscle attenuation group at the time of administrative censoring. Under these assumptions, a sample size of 120 at study commencement will provide $76 \%$ power to detect a difference in survivor functions between participants with low and normal muscle attenuation, with $\alpha=0.05$. Similarly, assuming $61 \%$ target population prevalence of low muscle mass ${ }^{20}$ and $50 \%$ survival in the normal muscle mass group, this sample size will provide $80 \%$ power to detect a minimum effect size of $\mathrm{HR}=2.06$ for the difference in survivor functions between participants with low and normal muscle mass.

\section{Statistical analysis}

All data will be analysed using the Stata software programme (StataCorp). Prior to formal data analysis, descriptive statistics and graphical displays will be used to identify missing and out-of-range values, assess the plausibility of means and SDs for all variables, identify outliers and screen continuous variables for normality. Recruitment bias will be assessed by comparing demographic and clinical variables for consenters with those who decline participation using t-tests (or Mann-Whitney U tests) for continuous variables and $\chi^{2}$ tests for categorical variables.

Modelling of study outcomes (eg, continuous scores, binary, counts) will be performed using generalised linear models with specification of appropriate distributions and link functions. For binary outcomes, the model that produces risk/rate ratios (RR), rather than ORs, (eg, binomial distribution with log link) will be used where possible due to the more intuitive interpretation of risk ratios when the outcome is common. ${ }^{51}$ Log-transformation of continuous outcome variables may be undertaken as indicated by model diagnostics. All regression analyses will be adjusted for study site and baseline levels of outcome variables (where available).

Prediction/multiple-exposure models will be built as follows: ${ }^{1}$ individual associations between exposure variables and the outcome will be tested in separate models; ${ }^{2}$ exposure variables showing at least weak evidence of an association with the outcome (defined here as $p$ value $<0.20$ ) in step 1 will be added to a multiple-exposure model; ${ }^{3}$ exposure variables in the multiple-exposure model with $p$ value $>0.10$ for their association with the outcome will be removed one at a time (in order of highest $\mathrm{p}$ value) and the model refitted until a final prediction model is determined. Given anticipated variability in time in treatment, models will be adjusted for treatment duration. 
Statistical assumptions, including linearity and (lack of) multicollinearity, will be examined and handled as necessary during the model-building process. The potential inflation of type I errors due to multiple testing will be taken into consideration when interpreting the results. Sensitivity analyses will be conducted to assess robustness of prediction models to influential observations if this appears warranted.

Overall survival will be defined as the number of days from the date of the baseline CT scan prior to CRT to the date of death by any cause or the date of censoring at 12 months following recruitment of the final participant. For participants still alive, overall survival will be censored at the last study visit, the last contact date or the date they were last known to be alive, whichever is last. Overall survival curves will be estimated using Kaplan-Meier methodology. Survival analysis will be conducted with Cox proportional hazards regression models.

The semistructured interviews will be recorded and content transcribed verbatim. Interview transcripts will be subject to thematic analysis. This will consist of inductive coding of textual data, interpreting using a constant comparative manner where concepts are labelled as codes. ${ }^{52}$ Codes will then be grouped into larger categories. An interrater process will be undertaken.

\section{Patient and public involvement}

A consumer representative was involved in this research from the time of the funding application where input was sought into the importance of the research question and expected outcomes from the study. During preparation of the study protocol, input was sought from our consumer representative regarding the wording and appropriateness of the qualitative interview questions, the participant information and consent form and the overall burden of participation. Consultation regarding dissemination of the study findings will occur with our consumer representative as well as a broader insight from a National Lung Cancer Patient Advisory Group.

\section{ETHICS AND DISSEMINATION}

The study received ethics approval from the Human Research Ethics Committee at Peter MacCallum Cancer Centre on 19 June 2019 (HREC/53147/PMCC-2019) and Deakin University (2019-320), and will be conducted in accordance with the principles of the Declaration of Helsinki.

The results of the study will be reported according to the Strengthening the Reporting of Observations Studies in Epidemiology guidelines. Dissemination of the findings will take the form of peer review publications and conference presentations.

\section{Author affiliations}

${ }^{1}$ Institute for Physical Activity and Nutrition, Deakin University, Geelong, Victoria, Australia

${ }^{2}$ Allied Health Department, Peter MacCallum Cancer Centre, Melbourne, Victoria, Australia

${ }^{3}$ School of Health Sciences, The University of Melbourne, Parkville, Victoria, Australia
${ }^{4}$ Department of Agricultural, Food and Nutrition Science, University of Alberta, Edmonton, Alberta, Canada

${ }^{5}$ Department of Radiation Oncology, Peter MacCallum Cancer Centre, Parkville, Victoria, Australia

${ }^{6}$ Sir Peter MacCallum Department of Oncology, University of Melbourne, Parkville, Victoria, Australia

${ }^{7}$ School of Nursing and Midwifery and Institute of Health Transformation, Deakin University, Geelong, Victoria, Australia

${ }^{8}$ Radiation Therapy Services, Peter MacCallum Cancer Centre, Parkville, Victoria, Australia

${ }^{9}$ Department of Medical Imaging and Radiation Therapy Services, Monash University, Clayton, Victoria, Australia

${ }^{10}$ Department of Physical Sciences, Peter MacCallum Cancer Centre, Parkville, Victoria, Australia

\section{Twitter Nicole K Kiss @NicoleKKiss and Sarah Everitt @EverittSJ}

Acknowledgements The authors thank ImpediMed, Australia, for the loan of the SOZO device used in this study and the Nutrition Department of Peter MacCallum Cancer Centre for assistance with PG-SGA training.

Contributors NKK, RMD, CMP, LD, LE contributed to conceptualisation. RMD, LD, CMP, LE, SFF, AU, SE, SS, DB, NH, GA, AW contributed to methodology. NKK, GA contributed to data analysis plan. NKK contributed to writing-original draft preparation. RMD, LD, CMP, LE, SFF, AU, SE, SS, DB, NH, GA, AW contributed to writing-review and editing. All authors approved the final version of the manuscript.

Funding NKK and this study are supported by a Victorian Cancer Agency Nursing and Allied Health Clinical Research Fellowship (grant no: CRFNAH18001).

Competing interests NKK reports grants from Victorian Cancer Agency, during the conduct of the study. AU reports grants from Victorian Cancer Agency, during the conduct of the study. RMD reports grants from World Cancer Research Fund International Regular Grant Programme, outside the submitted work. DB reports personal fees from Astra Zeneca, outside the submitted work. NH reports grants from Varian Medical Systems, outside the submitted work. SS reports personal fees from Astra Zeneca, outside the submitted work. CMP reports personal fees from Abbott Nutrition, Nutricia, Nestle Health Science, Fresenius Kabi and Pfizer, outside the submitted work. AW, GA, LD, LE and SE have nothing to disclose.

Patient consent for publication Not applicable.

Provenance and peer review Not commissioned; externally peer reviewed.

Supplemental material This content has been supplied by the author(s). It has not been vetted by BMJ Publishing Group Limited (BMJ) and may not have been peer-reviewed. Any opinions or recommendations discussed are solely those of the author(s) and are not endorsed by BMJ. BMJ disclaims all liability and responsibility arising from any reliance placed on the content. Where the content includes any translated material, BMJ does not warrant the accuracy and reliability of the translations (including but not limited to local regulations, clinical guidelines, terminology, drug names and drug dosages), and is not responsible for any error and/or omissions arising from translation and adaptation or otherwise.

Open access This is an open access article distributed in accordance with the Creative Commons Attribution Non Commercial (CC BY-NC 4.0) license, which permits others to distribute, remix, adapt, build upon this work non-commercially, and license their derivative works on different terms, provided the original work is properly cited, appropriate credit is given, any changes made indicated, and the use is non-commercial. See: http://creativecommons.org/licenses/by-nc/4.0/.

ORCID iDs

Nicole K Kiss http://orcid.org/0000-0002-6476-9834

Anna Ugalde http://orcid.org/0000-0002-2473-8435

Sarah Everitt http://orcid.org/0000-0001-6785-0067

\section{REFERENCES}

1 International Agency for Research on Cancer. Globocan 2018: estimated cancer incidence, mortality and prevalence in 2018 (lung cancer): World health organisation, 2019. Available: http://gco.iarc.fr/ today/data/factsheets/cancers/15-Lung-fact-sheet.pdf

2 Fearon K, Strasser F, Anker SD, et al. Definition and classification of cancer cachexia: an international consensus. Lancet Oncol 2011;12:489-95. 
3 Bradley JD, Paulus R, Komaki R, et al. Standard-Dose versus highdose conformal radiotherapy with concurrent and consolidation carboplatin plus paclitaxel with or without cetuximab for patients with stage IIIA or IIIB non-small-cell lung cancer (RTOG 0617): a randomised, two-by-two factorial phase 3 study. Lancet Oncol 2015;16:187-99.

4 Antonia SJ, Villegas A, Daniel D, et al. Durvalumab after chemoradiotherapy in stage III Non-Small-Cell lung cancer. N Engl J Med Overseas Ed 2017;377:1919-29.

5 Martin L, Birdsell L, Macdonald N, et al. Cancer cachexia in the age of obesity: skeletal muscle depletion is a powerful prognostic factor, independent of body mass index. J Clin Oncol 2013;31:1539-47.

6 Shen W, Punyanitya M, Wang Z, et al. Total body skeletal muscle and adipose tissue volumes: estimation from a single abdominal crosssectional image. J Appl Physiol 2004;97:2333-8.

7 Goodpaster BH, Kelley DE, Thaete FL, et al. Skeletal muscle attenuation determined by computed tomography is associated with skeletal muscle lipid content. J Appl Physiol 2000;89:104-10.

8 Prado CMM, Baracos VE, McCargar LJ, et al. Sarcopenia as a determinant of chemotherapy toxicity and time to tumor progression in metastatic breast cancer patients receiving capecitabine treatment. Clin Cancer Res 2009:15:2920-6.

9 Tan BHL, Birdsell LA, Martin L, et al. Sarcopenia in an overweight or obese patient is an adverse prognostic factor in pancreatic cancer. Clin Cancer Res 2009;15:6973-9.

10 Baracos VE, Reiman T, Mourtzakis M, et al. Body composition in patients with non-small cell lung cancer: a contemporary view of cancer cachexia with the use of computed tomography image analysis. Am J Clin Nutr 2010;91:1133S-7.

11 Prado CMM, Lieffers JR, McCargar LJ, et al. Prevalence and clinical implications of sarcopenic obesity in patients with solid tumours of the respiratory and gastrointestinal tracts: a population-based study. Lancet Oncol 2008:9:629-35.

12 Blauwhoff-Buskermolen S, Versteeg KS, de van der Schueren MAE, et al. Loss of muscle mass during chemotherapy is predictive for poor survival of patients with metastatic colorectal cancer. J Clin Oncol 2016;34:1339-44.

13 Antoun S, Baracos VE, Birdsell L, et al. Low body mass index and sarcopenia associated with dose-limiting toxicity of sorafenib in patients with renal cell carcinoma. Ann Oncol 2010;21:1594-8.

14 Prado CMM, Baracos VE, McCargar LJ, et al. Body composition as an independent determinant of 5-fluorouracil-based chemotherapy toxicity. Clin Cancer Res 2007;13:3264-8.

15 Antoun S, Lanoy E, lacovelli R, et al. Skeletal muscle density predicts prognosis in patients with metastatic renal cell carcinoma treated with targeted therapies. Cancer 2013;119:3377-84.

16 Ormsbee MJ, Prado CM, llich JZ, et al. Osteosarcopenic obesity: the role of bone, muscle, and fat on health. $J$ Cachexia Sarcopenia Muscle 2014;5:183-92.

17 Atlan P, Bayar MA, Lanoy E, et al. Factors which modulate the rates of skeletal muscle mass loss in non-small cell lung cancer patients: a pilot study. Support Care Cancer 2017;25:3365-73.

18 Lam VK, Bentzen SM, Mohindra P, et al. Obesity is associated with long-term improved survival in definitively treated locally advanced non-small cell lung cancer (NSCLC). Lung Cancer 2017;104:52-7.

19 Sheean PM, Peterson SJ, Gomez Perez S, et al. The prevalence of sarcopenia in patients with respiratory failure classified as normally nourished using computed tomography and subjective global assessment. JPEN J Parenter Enteral Nutr 2014;38:873-9.

20 Kiss N, Beraldo J, Everitt S. Early skeletal muscle loss in non-small cell lung cancer patients receiving chemoradiation and relationship to survival. Support Care Cancer 2019;27:2657-64.

21 Mourtzakis M, Prado CMM, Lieffers JR, et al. A practical and precise approach to quantification of body composition in cancer patients using computed tomography images acquired during routine care. Appl Physiol Nutr Metab 2008;33:997-1006.

22 Mitsiopoulos N, Baumgartner RN, Heymsfield SB, et al. Cadaver validation of skeletal muscle measurement by magnetic resonance imaging and computerized tomography. J Appl Physiol 1998;85:115-22.

23 Miller KD, Jones E, Yanovski JA, et al. Visceral abdominalfat accumulation associated with use of indinavir. Lancet 1998;351:871-5.

24 Morsbach F, Zhang Y-H, Martin L, et al. Body composition evaluation with computed tomography: contrast media and slice thickness cause methodological errors. Nutrition 2019:59:50-5.

25 Troschel AS, Troschel FM, Best TD, et al. Computed tomographybased body composition analysis and its role in lung cancer care. $J$ Thorac Imaging 2020;35:91-100.
26 Ottery FD. Definition of standardized nutritional assessment and interventional pathways in oncology. Nutrition 1996;12:S15-19.

27 Bauer J, Capra S, Ferguson M. Use of the scored Patient-Generated subjective global assessment (PG-SGA) as a nutrition assessment tool in patients with cancer. Eur J Clin Nutr 2002;56:779-85.

28 Isenring E, Bauer J, Capra S. The scored Patient-generated subjective global assessment (PG-SGA) and its association with quality of life in ambulatory patients receiving radiotherapy. Eur J Clin Nutr 2003;57:305-9.

29 Cederholm T, Jensen GL, Correia MITD, et al. GLIM criteria for the diagnosis of malnutrition - A consensus report from the global clinical nutrition community. Clin Nutr 2019;38:1-9.

30 National Cancer Institute. Dietary assessment primer: National cancer Institute. Available: https://dietassessmentprimer.cancer.gov/ profiles/table.html

31 Thompson FE, Subar AF. Dietary Assessment methodology. In: Coulston AM, Boushey CJ, Ferruzzi MG, et al, eds. Nutrition in the prevention and treatment of disease. Fourth Edition. Academic Press, 2017

32 Arends J, Bachmann P, Baracos V, et al. ESPEN guidelines on nutrition in cancer patients. Clin Nutr 2017;36:11-48.

33 Brown JC, Harhay MO, Harhay MN. Physical function as a prognostic biomarker among cancer survivors. Br J Cancer 2015:112:194-8.

34 Norman K, Stobäus N, Smoliner C, et al. Determinants of hand grip strength, knee extension strength and functional status in cancer patients. Clin Nutr 2010;29:586-91.

35 Schectman O, Sindhu BS. Grip assessment. In: Clinical assessment recommendations. Third ed. Mount Laurel, NJ: American Society of Hand Therapists, 2015.

36 Cruz-Jentoft AJ, Bahat G, Bauer J, et al. Sarcopenia: revised European consensus on definition and diagnosis. Age Ageing 2019;48:16-31

37 Malmstrom TK, Morley JE. SARC-F: a simple questionnaire to rapidly diagnose sarcopenia. J Am Med Dir Assoc 2013;14:531-2.

38 Barbosa-Silva TG, Menezes AMB, Bielemann RM, et al. Enhancing SARC-F: improving sarcopenia screening in the clinical practice. $J$ Am Med Dir Assoc 2016;17:1136-41.

39 Freedson PS, Melanson E, Sirard J. Calibration of the computer science and applications, Inc. accelerometer. Med Sci Sports Exerc 1998;30:777-81.

40 Matthews CE, Chen KY, Freedson PS, et al. Amount of time spent in sedentary behaviors in the United States, 2003-2004. Am J Epidemiol 2008;167:875-81.

41 Grant PM, Ryan CG, Tigbe WW, et al. The validation of a novel activity monitor in the measurement of posture and motion during everyday activities. Br J Sports Med 2006;40:992-7.

42 Grant PM, Dall PM, Mitchell SL, et al. Activity-monitor accuracy in measuring step number and cadence in community-dwelling older adults. J Aging Phys Act 2008;16:201-14.

43 Demeyer $\mathrm{H}$, Burtin C, Van Remoortel $\mathrm{H}$, et al. Standardizing the analysis of physical activity in patients with COPD following a pulmonary rehabilitation program. Chest 2014;146:318-27.

44 Craig CL, Marshall AL, Sjöström M, et al. International physical activity questionnaire: 12 -country reliability and validity. Med Sci Sports Exerc 2003;35:1381-95

45 Edbrooke L, Aranda S, Granger CL, et al. Benefits of home-based multidisciplinary exercise and supportive care in inoperable nonsmall cell lung cancer - protocol for a phase II randomised controlled trial. BMC Cancer 2017;17:663.

46 Butt Z, Webster K, Eisenstein AR, et al. Quality of life in lung cancer: the validity and cross-cultural applicability of the functional assessment of cancer Therapy-Lung scale. Hematol Oncol Clin North Am 2005;19:389-420.

47 Cella DF, Tulsky DS, Gray G, et al. The functional assessment of cancer therapy scale: development and validation of the general measure. J Clin Oncol 1993;11:570-9.

48 Forget $\mathrm{P}$, Khalifa C, Defour J-P, et al. What is the normal value of the neutrophil-to-lymphocyte ratio? BMC Res Notes 2017;10:12.

49 Creswell JW. Qualitative inquiry and research design. In: Oaks T, ed. Choosing among five approaches. 2nd edition. California: SAGE publications, 2007.

50 Kiss N, Isenring E, Gough K, et al. Early and Intensive Dietary Counseling in Lung Cancer Patients Receiving (Chemo) Radiotherapy-A Pilot Randomized Controlled Trial. Nutr Cancer 2016;68:958-67.

51 Persoskie A, Ferrer RA. A most odd ratio:: interpreting and describing odds ratios. Am J Prev Med 2017;52:224-8.

52 Eaves YD. A synthesis technique for grounded theory data analysis. J Adv Nurs 2001;35:654-63. 